\title{
Thermally induced self-agglomeration 3D scaffolds with BMP-2-loaded core-shell fibers for enhanced osteogenic differentiation of rat adipose-derived stem cells
}

This article was published in the following Dove Press journal:

International Journal of Nanomedicine

\author{
Shuying $\mathrm{Hu}^{\prime}$ \\ Hanbang Chen' \\ Xuefeng Zhou ${ }^{2}$ \\ Gang Chen' \\ $\mathrm{Ke} \mathrm{Hu}^{3}$ \\ Yi Cheng' \\ Lili Wang' \\ Feimin Zhang' \\ 'Jiangsu Key Laboratory of \\ Oral Diseases, Department of \\ Prosthodontics, Affiliated Hospital \\ of Stomatology, Nanjing Medical \\ University, Nanjing 210029 , \\ China; ${ }^{2}$ State Key Laboratory \\ of Bioelectronics, Jiangsu Key \\ Laboratory for Biomaterials and \\ Devices, School of Biological Science \\ and Medical Engineering, Southeast \\ University, Nanjing 210096, China; \\ ${ }^{3}$ Key Laboratory of Clinical and \\ Medical Engineering, Department of \\ Biomedical Engineering, School of \\ Basic Medical Science, Nanjing Medical \\ University, Nanjing 210000, China
}

Correspondence: Feimin Zhang Jiangsu Key Laboratory of Oral Diseases, Department of Prosthodontics, Affiliated Hospital of Stomatology, Nanjing Medical University, Nanjing 210029, China

Tel +86 258503 I83।

Fax +86 $25865164 \mid 4$

Email fmzhang@njmu.edu.cn

\begin{abstract}
Introduction: Scaffold structure plays a vital role in cell behaviors. Compared with two-dimensional structure, 3D scaffolds can mimic natural extracellular matrix (ECM) and promote cell-cell and cell-matrix interactions. The combination of osteoconductive scaffolds and osteoinductive growth factors is considered to have synergistic effects on bone regeneration.

Materials and methods: In this study, core-shell poly(lactide-co-glycolide) (PLGA)/ polycaprolactone (PCL)-BMP-2 (PP-B) fibrous scaffolds were prepared through coaxial electrospinning. Next, we fabricated 3D scaffolds based on PP-B fibers with thermally induced self-agglomeration (TISA) method and compared with conventional PLGA/PCL scaffolds in terms of scaffold morphology and BMP-2 release behaviors. Then, rat adipose-derived stem cells (rADSCs) were seeded on the scaffolds, and the effects on cell proliferation, cell morphology, and osteogenic differentiation of rADSCs were detected.
\end{abstract}

Results: The results demonstrated that 3D scaffold incorporated with BMP-2 significantly increased proliferation and osteogenic differentiation of rADSCs, followed by PP-B group.

Conclusion: Our findings indicate that scaffolds with 3D structure and osteoinductive growth factors have great potential in bone tissue engineering.

Keywords: BMP-2, core-shell scaffolds, 3D fibrous scaffolds, osteogenic differentiation

\section{Introduction}

Dental implants are gaining great popularity in repairing dentition defects. ${ }^{1}$ However, many patients fail to accept the implant treatment because of bone insufficiency. Also, bone defects resulting from accidents, infection, and tumors require large amount of bone grafts, which are relatively in shortage in clinical practice., ${ }^{2,3}$ As a result, bone tissue engineering is developing as an alternative approach to solve these problems. ${ }^{4}$

Electrospinning has emerged as a promising method to fabricate scaffolds in tissue engineering in recent decades. ${ }^{5,6}$ Electrospun scaffolds with micro- to nanoscale fibers have shown excellent mechanical properties and biocompatibilities, thanks to their high porosity and high surface area-to-volume ratios, as well as the resemblance of extracellular matrix (ECM). ${ }^{7}$ All of these merits make electrospun scaffolds an effective approach to promote cell-matrix communication ${ }^{8}$ and facilitate bone regeneration., ${ }^{9}, 10$ Recent studies have exhibited enhanced osteogenic differentiation by incorporating growth factors into scaffolds. ${ }^{11,12}$

Bone morphogenetic protein 2 (BMP-2) is one of the most potent inducers in bone regeneration ${ }^{13}$ and has strong osteoinduction capability in osteogenic differentiation 
of mesenchymal stem cells (MSCs). ${ }^{14} \mathrm{Su}$ et al ${ }^{15}$ reported a significantly enhanced osteoblast differentiation of MSCs induced by core/shell poly(L-lactide-co-caprolactone)/ collagen scaffolds loaded with BMP-2 and dexamethasone through coaxial electrospinning. Coaxial electrospinning is a method that allows two different solutions electrospun simultaneously to produce fibers with core-shell structures. Bioactive proteins in core can be protected by shell and released in a sustained and controlled manner, ${ }^{16}$ thus enabling a certain concentration of bioactive proteins to be maintained in target area.

Owing to the above superiority, coaxial electrospinning was used in our study to fabricate core-shell fibers loaded with BMP-2. However, conventional electrospun scaffolds with two-dimensional (2D) structure at macroscale are of small pore size and poor interconnectivity, which restricts cell infiltration and migration. ${ }^{17}$ Cellular infiltration is important to construct 3D cell-scaffold complex and facilitate integration with host tissue after implantation. ${ }^{18}$ Scaffold architecture has great impacts on cellular behaviors through cell-material interaction. ${ }^{19,20}$ 3D scaffolds are able to provide stronger cell-cell interactions than 2D structure. ${ }^{21}$ In fact, it has been reported that stem cells cultured on 3D scaffolds exhibited enhanced osteogenic differentiation ability. ${ }^{22}$ In light of these advantages, great efforts have been made to fabricate 3D porous scaffolds based on electrospinning for enhanced biological performance. For instance, Yao et $\mathrm{al}^{23}$ fabricated 3D porous polycaprolactone (PCL)/polylactic acid (PLA) scaffolds by combining electrospun fibers with TISA method. The scaffolds were validated to facilitate stem cell differentiation and bone regeneration through in vitro and in vivo studies.

Poly(lactide-co-glycolide) (PLGA) and PCL are widely used in bone tissue engineering for their excellent mechanical properties and biocompability. ${ }^{24,25}$ In fact, PLGA and PCL are approved by FDA for biomedical applications. ${ }^{19,26}$ Our previous study confirmed that scaffolds made of PLGA and PCL facilitated osteogenic differentiation of rat adiposederived stem cells (rADSCs). ${ }^{27}$

ADSCs have aroused more attention in tissue engineering due to its easy access to large amounts and relatively noninvasive acquisition method. ${ }^{28,29}$ Scaffolds seeded with rADSCs significantly promoted bone regeneration after implantation in rat critical-sized calvarial defects when compared with pure scaffolds. ${ }^{30}$

In this study, we fabricated PLGA/PCL scaffolds loaded with BMP-2 through coaxial electrospinning. Then, TISA technique was used to construct $3 \mathrm{D}$ porous scaffolds. Cellular behaviors were studied to evaluate the effects of BMP-2 and $3 \mathrm{D}$ structure on the osteogenic differentiation of rADSCs.

\section{Materials and methods}

PLGA (75:25, MW=120,000 Da) and PCL (MW=120,000 Da) were obtained from Daigang Biomaterial Co., Ltd (Jinan, China). 2,2,2-Trifluoroethanol (TFEA) was purchased from Aladdin Biochemical Technology Co., Ltd (Shanghai, China). BMP-2 was supplied by Peprotech (Rocky Hill, NJ, USA). Enzyme-linked immunosorbent assay (ELISA) kits for BMP-2 was purchased from Boster Biological Technology Co., Ltd (Wuhan, China). Cell counting kit-8 (CCK-8; Biosharp, Hefei, China), 0.25\% Trypsin-EDTA (Thermo Fisher Scientific, Waltham, MA, USA), bicinchoninic acid protein assay kit (Leagene Biotechnology, Beijing, China), alkaline phosphatase (ALP) assay kit (Jiancheng Bioengineering Institute, Nanjing, China), BCIP/NBT Alkaline Phosphatase Color Development Kit (Beyotime Biotechnology, Shanghai, China), and Alizarin red S (ARS; Leagene Biotechnology, Beijing, China) were used in this study. OriCell Sprague Dawley (SD) rADSCs, SD Rat Adipose-derived Stem Cell Osteogenic Differentiation Medium, and SD Rat Adipose-derived Mesenchymal Stem Cell Complete Medium were purchased from Cyagen Biosciences (Guangzhou, China). The osteogenic differentiation medium consisted of $175 \mathrm{~mL}$ of basal medium, $20 \mathrm{~mL}$ of fetal bovine serum, $2 \mathrm{~mL}$ of penicillin-streptomycin, $2 \mathrm{~mL}$ of glutamine, $400 \mu \mathrm{L}$ of ascorbate, $2 \mathrm{~mL}$ of $\beta$-glycerophosphate, and $20 \mu \mathrm{L}$ of dexamethasone. Takara Minibest universal RNA Extaction Kit, PrimerScript RT Master Mix, and SYBR Premix EX Taq II were supplied by Takara Bio Inc (Kusatsu, Japan).

\section{Preparation of electrospun scaffolds}

Core-shell PP-BMP-2 (PP-B) electrospun scaffolds and conventional PP electrospun scaffolds were fabricated. Figure 1 demonstrates the preparation of core-shell scaffold. To fabricate core-shell PP-B electrospun scaffolds, PLGA and PCL ( $0.75 \mathrm{~g}$ of each) were dissolved in $8.5 \mathrm{~g}$ of TFEA and mixed by magnetic stirrer overnight to produce $15 \%(\mathrm{w} / \mathrm{w})$ shell solution. To prepare core solution, BMP-2 $(10 \mu \mathrm{g} / \mathrm{mL})$ was dissolved in distilled deionized water with bovine serum albumin (BSA) (1 mg/mL; Solarbio Life Science, Beijing, China) as a protein stabilizer. The solution was put into two syringes connected with a coaxial spinneret (inner needle: 22 G; outer needle: 17 G; Figure 1) to produce fibers with core-shell structure. A high-voltage power supply (Dong Wen, Tianjin, China) was used. The core solution was injected at a flow rate of $0.4 \mathrm{~mL} / \mathrm{h}$, and the shell solution was injected at a flow rate of $2.0 \mathrm{~mL} / \mathrm{h}$. An applied voltage of $20 \mathrm{kV}$ and a tip-to-collector distance of $20 \mathrm{~cm}$ were set to fabricate fibrous scaffolds with coreshell structure. 
A
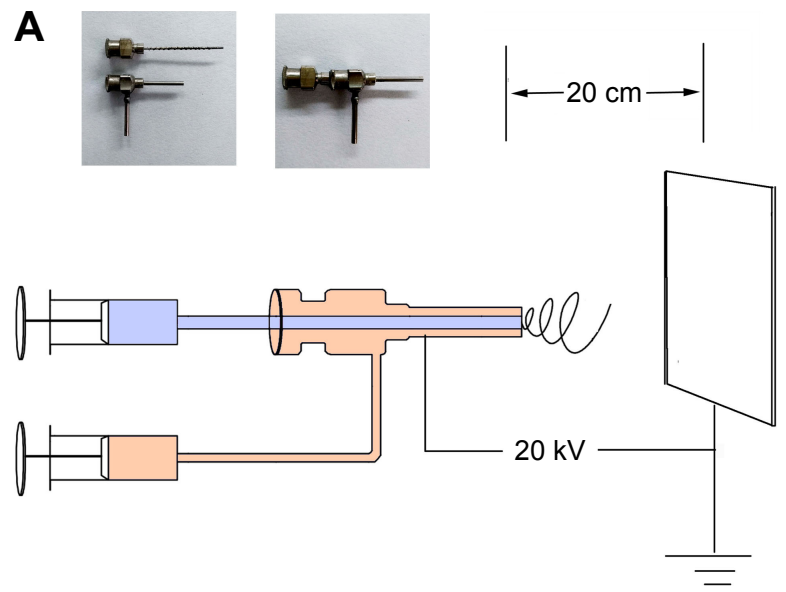

B

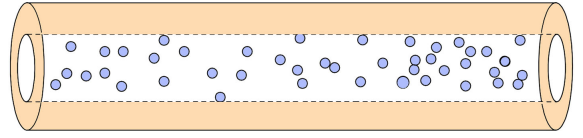

Figure I Illustrations of electrospun scaffolds' preparation.

Notes: (A) Schematic drawing of coaxial electrospinning process. Inset: coaxial spinneret. (B) Schematic drawing of coaxial electrospinning fibers (core: BMP-2, shell: PLGA/PCL).

Abbreviations: BMP-2, bone morphogenetic protein 2; PCL, polycaprolactone; PLGA, poly(lactide-co-glycolide).

PP electrospun scaffolds were prepared as control group. Briefly, PLGA and PCL mixture solution was fabricated using the same concentration as mentioned earlier. The solution was then electrospun with a high voltage of $20 \mathrm{kV}$, a flow rate of $0.4 \mathrm{~mL} / \mathrm{h}$, and a tip-to-collector distance of $20 \mathrm{~cm}$.

The thickness of the fibrous scaffolds was $\sim 150 \mu \mathrm{m}$. The samples were thoroughly freeze-dried for $24 \mathrm{~h}$. The PP-B scaffolds and PP scaffolds were stored at $-20^{\circ} \mathrm{C}$ for later use.

\section{Fabrication of 3D scaffolds}

3D scaffolds were fabricated using the TISA technique. ${ }^{31}$ First, $65 \mathrm{mg}$ of electrospun PP-B scaffolds were cut into small pieces $(0.2 \times 0.2 \mathrm{~cm})$ and suspended uniformly in $2 \mathrm{~mL}$ of gelatin aqueous solution using a homogenizer (FSH-2A; Friend Instrument, Changzhou, China) with the revolving speed of 10,000 rpm for $5 \mathrm{~min}$. The fiber suspension was transferred into a glass bottle. The bottle was immediately immersed in water at $55^{\circ} \mathrm{C}$ for $2 \mathrm{~min}$. During this period, the small pieces spontaneously agglomerated into a $3 \mathrm{D}$ scaffold with $12-14 \mathrm{~mm}$ in diameter and $2 \mathrm{~mm}$ in thickness. Then, the $3 \mathrm{D}$ scaffold was put in ice water for $2 \mathrm{~min}$ to prevent further shrinkage. Finally, the 3D scaffolds were freeze-dried for $24 \mathrm{~h}$ and kept at $-20^{\circ} \mathrm{C}$.

\section{Scanning electron microscopy (SEM) and transmission electron microscopy (TEM)}

The morphology of electrospun scaffolds and 3D scaffolds was observed by an SEM (S-3400N II; Hitachi Ltd., Tokyo, Japan) after gold coating at an accelerating voltage of $20 \mathrm{kV}$. The fiber diameter and pore size were calculated using the
ImageJ software (National Institutes of Health, Bethesda, MD, USA).

For cell morphology observation, rADSCs were seeded on the scaffolds and cultured for 7 days. Then, the samples were fixed with $2.5 \%$ glutaraldehyde, dehydrated, freezedried, and imaged by SEM.

The core-shell structure of PP-B fibers was detected by TEM (JEM-2100; JEOL, Tokyo, Japan) after being collected on a copper grid and vacuum dried. Shell thickness and core diameter were calculated by measuring randomly selected fibers using the ImageJ software.

\section{Fluorescent microscopy}

In order to visualize the distribution of BMP-2 in core-shell fibers, FITC-BSA (Solarbio Life Science) was used as a replacement for BMP-2 to fabricate PP-FITC-BSA coreshell fibers with PP in shell and FITC-BSA in core under the same condition. Several fibers were collected on a glass slide and examined under a fluorescent microscope (DM4000; Leica Microsystems, Wetzlar, German).

\section{ELISA}

In vitro BMP-2 release from PP-B scaffold and 3D scaffold was detected by ELISA kit. PP-B scaffold and 3D scaffold were sterilized with UV light and washed gently with PBS for three times. Then, the samples were put into a 24-well plate with $1 \mathrm{~mL}$ of PBS and placed in an incubator $\left(37^{\circ} \mathrm{C}, 5 \% \mathrm{CO}_{2}\right)$. The extracts were collected at specific time point $(1,3,5,7,14,21$, and 28 days $)$, and $1 \mathrm{~mL}$ of PBS was added to each well for further release. The supernatants were stored at $-80^{\circ} \mathrm{C}$ until all the samples were collected. The amount of BMP-2 released was measured using an ELISA kit following the manufacturing's instruction. 


\section{Cell culture and sample preprocessing}

SD rADSCs were cultured in a cell incubator at $37^{\circ} \mathrm{C}$ at a humidified atmosphere containing $5 \% \mathrm{CO}_{2}$. The rADSCs were passaged after reaching $\sim 80 \%$ confluence. Cells at passages 3-5 were used in our experiments. Before cell seeding, the scaffolds were sterilized with $75 \%$ ethanol for $2 \mathrm{~h}$, washed with PBS twice, and then precultured in the culture medium for $3 \mathrm{~h}$ at $37^{\circ} \mathrm{C}$. To ensure most cells adhere on the scaffold rather than on the well plate, $50 \mu \mathrm{L}$ of cell suspension of $5 \times 10^{4}$ rADSCs was seeded on each scaffold in 24-well plate. After incubating for $4 \mathrm{~h}, 1 \mathrm{~mL}$ of culture medium was added in each well. The medium was changed every 2 days.

\section{Cell proliferation analysis}

For proliferation analysis, the rADSCs were seeded at 5,000 cells/scaffold (6 mm in diameter) in 96-well plate. CCK-8 was used to measure cell viability at days $1,3,5$, and 7 after cell seeding according to manufacturer's instructions. In brief, CCK-8 solution was mixed with culture medium and then added to each well. After being incubated for $2 \mathrm{~h}$ at $37^{\circ} \mathrm{C}$, all samples were measured using a microplate reader (Spectra Max 190; Molecular Devices LLC, Sunnyvale, CA, USA) at the absorbance of $450 \mathrm{~nm}$.

\section{Confocal laser scanning microscopy (CLSM) observation}

Each scaffold was seeded with $2 \times 10^{4}$ rADSCs in 24-well plate. The morphology of rADSCs adhering on the scaffolds was observed by CLSM (Zeiss-LSM710; Carl Zeiss Meditec AG, Jena, Germany). The methods were reported in our previous study. ${ }^{32}$ In brief, at day 1, cells were permeabilized using $0.5 \%$ Triton X-100 (Solarbio Life Science) for $10 \mathrm{~min}$ and then stained with rhodamine phalloidin (Cytoskeleton, Inc., Denver, CO, USA) and 4',6-diamidino-2-phenylindole (DAPI; Beyotime Biotechnology) to label F-actin and cell nuclei, respectively.

\section{ALP activity assay and ALP staining}

The rADSCs were seeded at $5 \times 10^{4}$ cells/scaffold in 24 -well plate and incubated in osteogenic medium. After osteoinduction for 7 and 14 days, rADSCs in different groups were lysed with $0.5 \%$ TritonX-100 for $30 \mathrm{~min}$. Then, the supernatant was collected for analysis. ALP activity and total protein concentration were detected according to the manufacturer's instructions of ALP assay kit and bicinchoninic acid protein assay kit, respectively. ALP activity levels were normalized to the total protein content.
For ALP staining, cells on the scaffolds were stained following the manufacturer's instructions of ALP staining kit. Briefly, the samples were fixed with $4 \%$ paraformaldehyde and washed with PBS for twice. Then, the scaffolds were stained with BCIP/NBT reagents and washed with double distilled water for observation.

\section{Matrix mineralization assay}

Matrix mineralization level was evaluated by Alizarin red staining. After osteogenic induction of 14 days, rADSCs on the samples were fixed with $4 \%$ paraformaldehyde and then stained with $1 \%$ ARS (pH 4.2) for 5 min. After qualitative observation, the samples were washed with PBS and extracted with $10 \%$ cetylpyridinium chloride (Bomei Biotechnology, Hefei, China) for $15 \mathrm{~min}$ at room temperature to detect calcium nodules. Then, the solution was transferred to a 96-well plate for absorbance reading at $550 \mathrm{~nm}$ using a microplate reader.

\section{Real-time quantitative reverse transcription polymerase chain reaction (RT-PCR) analysis of osteogenic differentiation markers}

RT-PCR was performed to evaluate the mRNA expression level of osteogenic differentiation markers of Alp, Coll, $M s x 2$, and $O c n$ in the three scaffolds after osteogenic induction for 7 and 14 days. Total RNA was extracted using the RNA extraction kit following the manufacturer's protocol. A total of 500 ng of RNA was reverse transcribed into cDNA with a reverse transcription kit, and real-time PCR was performed using the ABI 7300 Real-Time PCR System (Thermo Fisher Scientific). The primer sequences are listed in Table 1. Relative expression level of target gene was normalized to GAPDH and calculated using the $2^{-\Delta \Delta c t}$ method. The experiment was performed for three times independently.

\section{Statistical analysis}

All data were shown as mean \pm standard deviation (SD). Statistical analysis was performed using the SPSS v.19.0 software (IBM Corporation, Armonk, NY, USA). Quantitative data were analyzed using one-way ANOVA followed by the least significant difference (LSD) test. Statistical significance was set at the level of $P<0.05$.

\section{Results and discussion Morphology of the scaffolds}

The SEM images of the three groups are shown in Figure 2. PP and PP-B fibers showed smooth and beads free surface 
Table I Primer sequences for RT-PCR analysis

\begin{tabular}{lll}
\hline Gene & Forward primer $\left(\mathbf{5}^{\prime} \mathbf{-} \mathbf{3}^{\prime} \mathbf{)}\right.$ & Reverse primer $\left(\mathbf{5}^{\prime} \mathbf{- 3} \mathbf{3}^{\prime}\right)$ \\
\hline Alp & CATGTTCCTGGGAGATGGTATG & GTGTTGTACGTCTTGGAGAGAG \\
Coll & TGTTGGTCCTGCTGGCAAGAATG & GTCACCTTGTTCGCCTGTCTCAC \\
Ms 2 & CCTGCACCCTGAGGAAACAC & TGCCTTAGCCCTTCGGTTCT \\
Ocn & ACCCTCTCTCTGCTCACTCTGC & CCTTACTGCCCTCCTGCTTGG \\
GAPDH & GGGAAACCCATCACCATCTT & CCAGTAGACTCCACGACATACT \\
\hline
\end{tabular}

Abbreviation: RT-PCR, real-time quantitative reverse transcription polymerase chain reaction.

with uniform diameters (Figure 2A and B). The core-shell structure of PP-B fibers was displayed in TEM image (Figure 2D and E). Image analysis showed that the average fiber diameters for PP and PP-B were 806.5 \pm 129.4 and $760.1 \pm 144.3 \mathrm{~nm}$, respectively. The pore sizes of PP, PP-B, and 3D scaffolds were $9.95 \pm 1.78,9.14 \pm 1.78$, and $80.95 \pm 12.43 \mu \mathrm{m}$, respectively. The core diameter of coreshell fibers in PP-B scaffold was $383.49 \pm 53.50 \mathrm{~nm}$ and the shell thickness was $80.37 \pm 19.35 \mathrm{~nm}$. The TEM images and measurement results indicated that PP-B fibers owned thin shell thickness. Previous study reported that silk fibroin/ chitosan/nanohydroxyapatite/BMP-2 core-shell nanofibrous scaffold with thin shell thickness exhibited a higher concentration of BMP-2 in release medium and a stronger osteogenic effect both in vitro and in vivo. ${ }^{33}$ Figure $2 \mathrm{~F}$ is the fluorescent microscopic image of PP-FITC-BSA core-shell fibers. Coaxial electrospun FITC-BSA formed a line-like structure when observed under a fluorescent microscope, which confirmed the continuous distribution of FITC-BSA in core. Through coaxial electrospinning, BMP-2 was protected from direct contact with organic solvent and remained its osteogenic activity. ${ }^{34}$

Figure 2C demonstrates the SEM micrograph of 3D scaffold. Fibers in 3D scaffold were not damaged during preparation and connected with each other to form large and interconnected pores. The porous structure in the scaffold mimics natural ECM and provides a pathway for cell migration, as well as a passage for nutritional metabolism

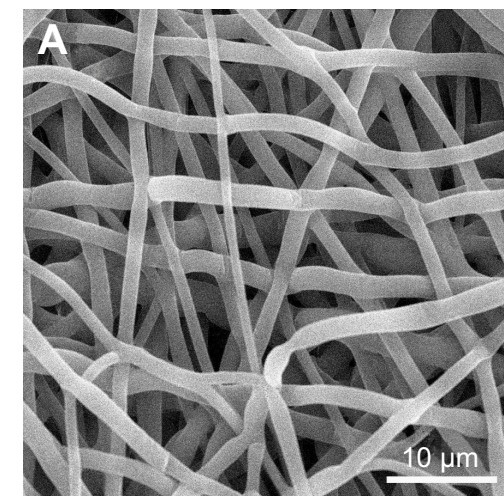

D
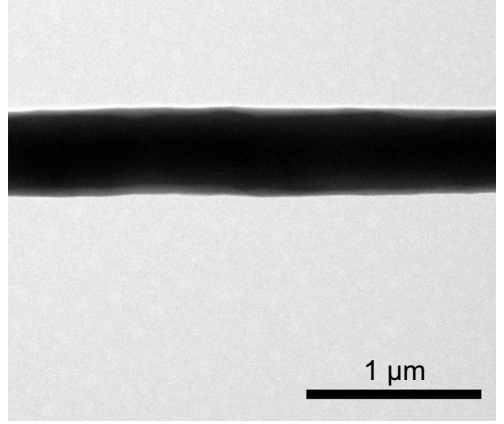

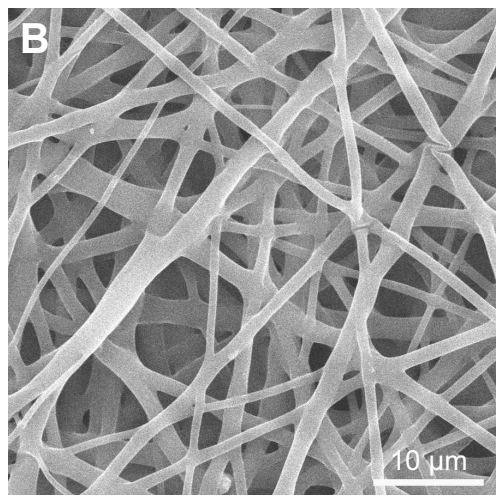

E

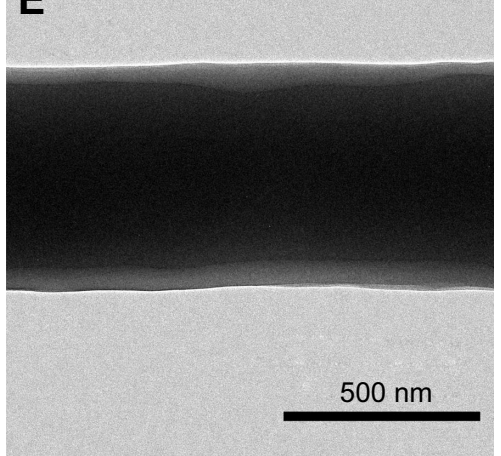

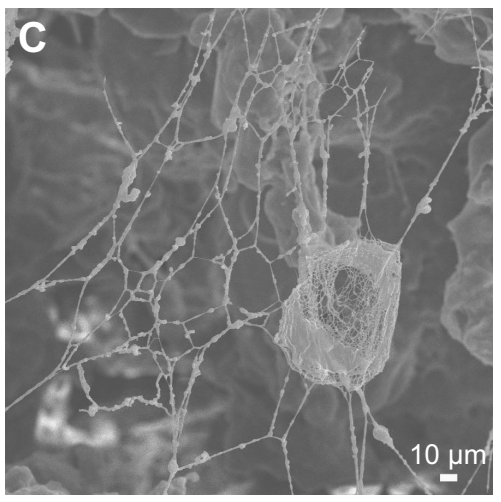

$\mathbf{F}$

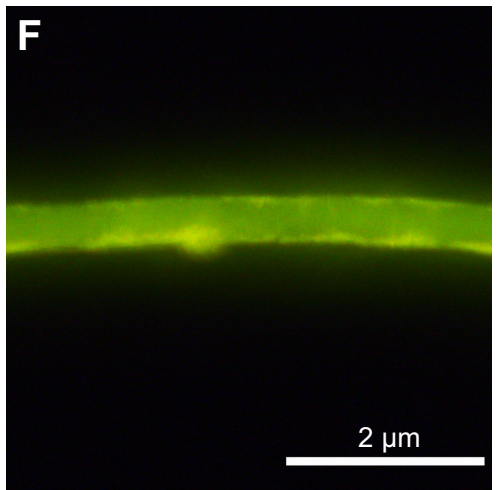

Figure 2 Characterization of different scaffolds.

Notes: SEM images of the following three scaffolds: PP (A), PP-B (B), and 3D (C). TEM images of PP-B fibers (D and E). Fluorescent microscopic image of PP-FITC-BSA core-shell fiber (F).

Abbreviations: 3D, three dimension; PCL, polycaprolactone; PLGA, poly(lactide-co-glycolide); PP, PLGA/PCL; PP-B, PP-bone morphogenetic protein 2; SEM, scanning electron microscopy; TEM, transmission electron microscopy. 
and gas exchange, which is beneficial for cells' growth and tissue regeneration. ${ }^{35,36}$

\section{In vitro BMP-2 release evaluation}

Figure 3 illustrates in vitro cumulative release behaviors of BMP-2 in PP-B and 3D scaffolds. Both scaffolds showed a sustained release of BMP-2 within 28 days with an initial burst at the first day. PP-B scaffold exhibited an obviously higher level of BMP-2 than 3D group at each examined time point.

In a sense, electrospinning is an unstable process; therefore, part of BMP-2 attached to the surface instead of being completely encapsulated into "core-shell" fibers. ${ }^{16}$ This may explain the relatively high concentration of BMP-2 released at day 1 in PP-B samples. BMP-2 was released in a stable manner afterward. In 3D groups, BMP-2 was partially lost during immersion in gelatin aqueous solution for several minutes. As a result, the amount of BMP-2 released from 3D scaffold was significantly lower than that of PP-B scaffold.

\section{Cell proliferation}

Cell proliferation on different scaffolds was examined by CCK-8 (Figure 4). The optical density (OD) value of all groups gradually increased from days 1 to 7 . This demonstrated that rADSCs were able to attach and proliferate well on all the three scaffolds. At day 1 , the number of rADSCs in 3D scaffold was significantly higher than the other two groups $(P<0.05)$, indicating a higher initial attachment of cells on $3 \mathrm{D}$ group. Scaffold structures play an important role in cell behavior, such as cell proliferation and cell differentiation. ${ }^{37}$

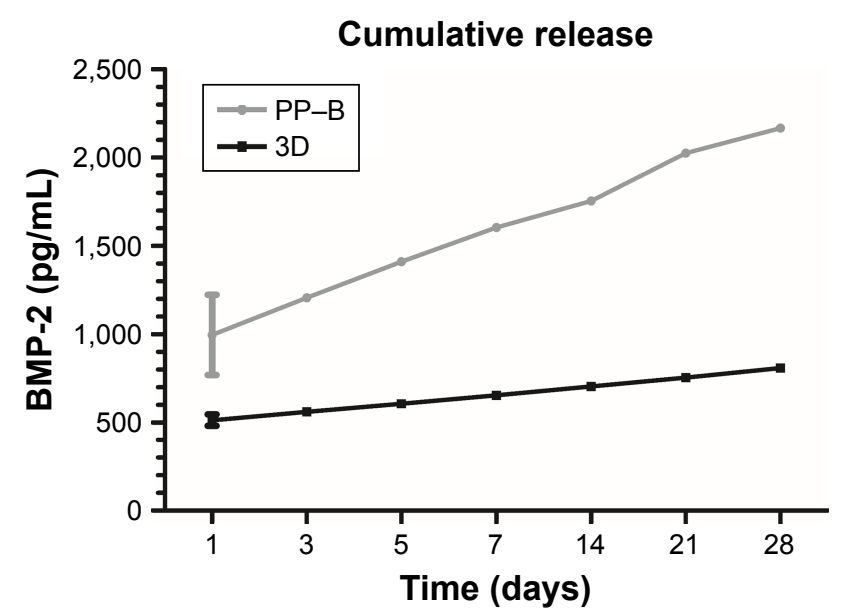

Figure 3 Cumulative release curves of BMP-2 from PP-B scaffold and 3D scaffold within 28 days.

Abbreviations: 3D, three dimension; BMP-2, bone morphogenetic protein-2; PP-B, poly(lactide-co-glycolide)/polycaprolactone-BMP-2.

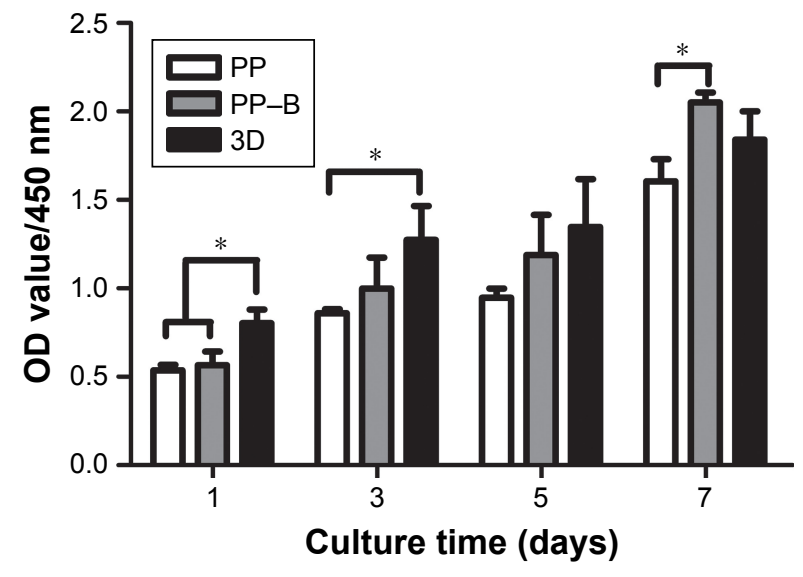

Figure 4 Proliferation of rADSCs on scaffolds with CCK-8 assay. Note: $* P<0.05$.

Abbreviations: 3D, three dimension; CCK-8, cell counting kit-8; PP, poly(lactideco-glycolide)/polycaprolactone; PP-B, PP-bone morphogenetic protein-2; rADSCs, rat adipose-derived stem cells.

3D scaffold had larger surface areas, and this could contribute to the increased cellular adhesion at day 1 .

There was no significant difference in cell proliferation between PP and PP-B until 5 days. This result could be ascribed to the excellent biocompatibility of the three scaffolds. These three scaffolds exhibited excellent biological properties, so the addition of BMP-2 was unable to cause significant differences. However, a longer culture time would increase the accumulation of BMP-2, which caused the better proliferation of PP-B group at day 7.

After 7 days, higher numbers of rADSCs were found on PP-B scaffold than on PP scaffold $(P<0.05)$, while no statistical difference of OD value was observed between $\mathrm{PP}-\mathrm{B}$ group and 3D group. Our finding is consistent with $\mathrm{Fu}$ et al' ${ }^{38}$ research, which revealed that immobilized BMP-2 on microcarriers improved cell proliferation. Osteogenic differentiation usually began after reaching a certain confluence. This may explain why cell proliferation slowed down in 3D group after 7 days. Our result demonstrated no difference of cell proliferation between PP-B scaffold and 3D scaffold at day 7. However, some studies found better proliferation on 3D scaffolds than on 2D scaffolds. Cell number maintained the same when reaching the maximum value in $3 \mathrm{D}$ matrices, but the number reduced after confluence in $2 \mathrm{D}$ matrices resulting from cell detaching from $2 \mathrm{D}$ surface. ${ }^{37} 3 \mathrm{D}$ structure is able to provide more space for cell growth, and superior proliferation ability is a promising factor for osteogenic differentiation.

\section{Cell morphology on the scaffolds}

Cell morphology was evaluated by CLSM and SEM. Cytoskeleton (red) and cell nuclei (blue) were visualized by staining 
with rhodamine phalloidin and DAPI after culturing for 1 day (Figure 5A-C). Cells showed a relatively round shape in PP scaffold while exhibited a polygon morphology with stretched cytoskeleton in PP-B scaffold. Cell aggregates formed and thereafter were able to fully extend to spatial area in 3D scaffold.

SEM images of cells grown on the three scaffolds are shown in Figure 5D-F. The rADSCs were well spreaded in all scaffolds. In PP and PP-B groups, cells exhibited a flattened morphology. In 3D group, cells formed a 3D structure thanks to the sufficient space, which indicated that $3 \mathrm{D}$ porous fibrous scaffolds promoted cell adhesion and cell stretching than 2D surface.

Cell migration and cellular shapes were greatly affected by scaffold structures through cell-material interactions. ${ }^{39}$ Hong et $\mathrm{al}^{40}$ reported that most endothelial progenitor cells migrated through $3 \mathrm{D}$ scaffolds while tended to stay on the surface of 2D scaffolds.

\section{ALP activity and mineralization evaluation}

ALP, as a marker of early osteoblastic differentiation, ${ }^{41}$ was selected to indicate the osteogenic differentiation level of rADSCs in this study. The results of ALP activity and ALP staining after the osteogenic induction of 7 and 14 days are shown in Figure 6. PP-B and 3D groups expressed a higher level of ALP activity than PP group, while 3D group was the highest, both after osteogenic induction for 7 and 14 days. The ALP activity increased from days 7 to 14 in all groups, which was consistent with the results of ALP staining assay. This suggested the positive effects of BMP-2 and 3D structure on the osteogenic differentiation of rADSCs.

Alizarin red staining was performed to evaluate calcium nodules in the three scaffolds after osteogenic induction for 14 days (Figure 6C). Calcium deposition on 3D scaffold was obviously higher than the other two groups, while the lowest mineralization level was seen in PP group. The result was confirmed by the semiquantification examination of ARS extracts (Figure 6D).

A higher level of ALP activity and calcification degree in $\mathrm{PP}-\mathrm{B}$ scaffold was caused by the sustained release of BMP-2. BMP-2 can facilitate osteogenic differentiation of mesenchymal cells through Smad signal pathway. ${ }^{42}$ Previous study demonstrated that BMP-2 was able to accelerate calcification and bone regeneration. ${ }^{43}$ Liu et $\mathrm{al}^{44}$ reported that nanocomplexes loaded with BMP-2 significantly promoted ALP activity of MC3T3-E1 cells with an increased mineralization level of ECM.

In 3D scaffold, synergistic effects of BMP-2 and physical-mechanical characteristics of 3D scaffold could
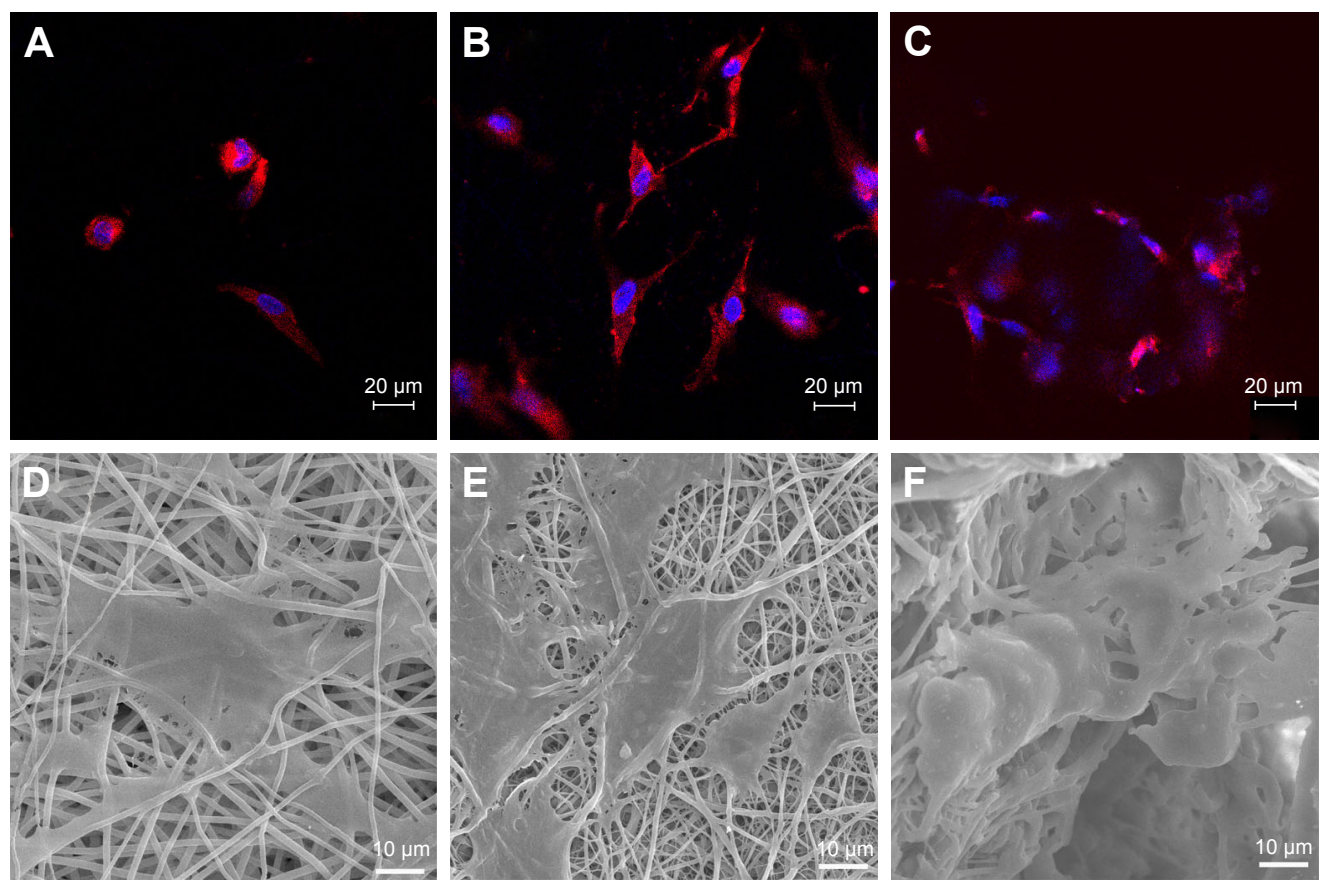

Figure 5 Morphology of rADSCs on scaffolds.

Notes: Representative CLSM images of rADSCs on scaffolds after I day: PP (A), PP-B (B), 3D (C), cytoskeleton (red), and cell nuclei (blue). Representative SEM images of ADSCs on scaffolds after 7 days: PP (D), PP-B (E), and 3D (F).

Abbreviations: 3D, three dimension; CLSM, confocal laser scanning microscopy; PP, poly(lactide-co-glycolide)/polycaprolactone; PP-B, PP-bone morphogenetic protein-2; rADSCs, rat adipose-derived stem cells; SEM, scanning electron microscopy. 
A
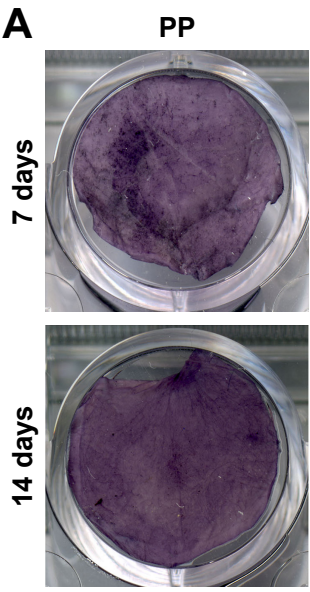

C

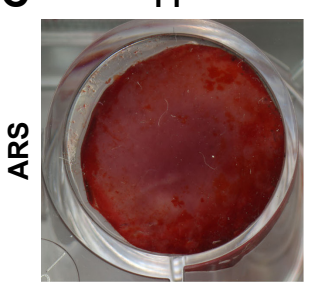

PP-B

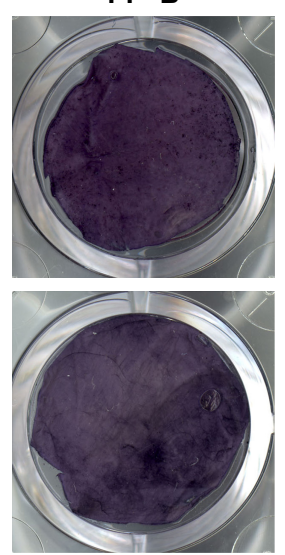

PP-B

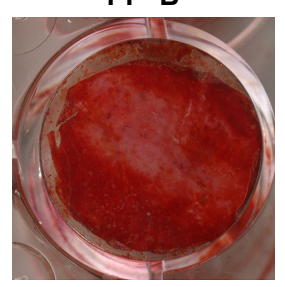

3D

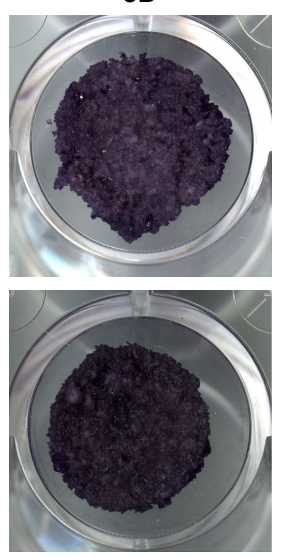

3D

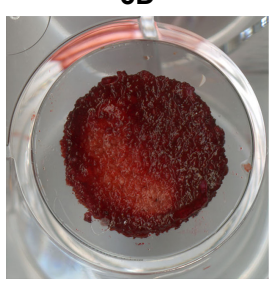

B

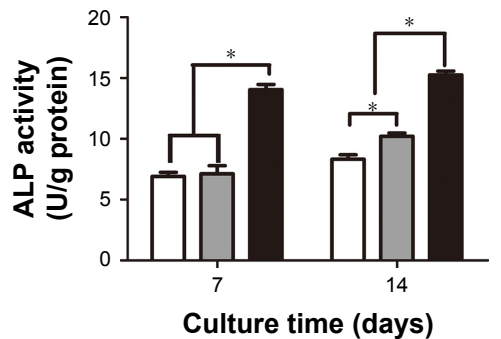

Culture time (days)

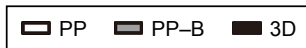

D

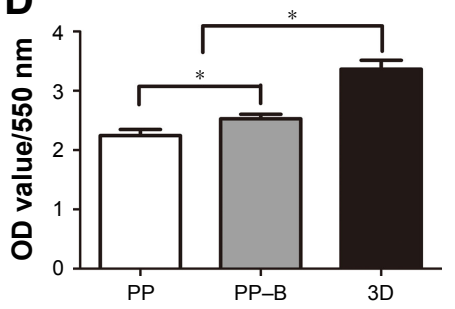

Figure 6 ALP activity of rADSCs and calcium deposition evaluation on the scaffolds.

Notes: (A) ALP staining of rADSCs on the scaffolds after osteogenic induction of 7 and 14 days. (B) ALP activity of rADSCs on different scaffolds after osteogenic induction of 7 and 14 days $(* P<0.05)$. (C) Alizarin red staining of rADSCs on the three scaffolds after osteogenic induction of 14 days. (D) Semiquantitative analysis of calcium deposition on the scaffolds after osteogenic induction of 14 days for rADSCs $(* P<0.05)$.

Abbreviations: 3D, three dimension; ALP, alkaline phosphatase; ARS, Alizarin red S; PP, poly(lactide-co-glycolide)/polycaprolactone; PP-B, PP-bone morphogenetic protein-2; rADSCs, rat adipose-derived stem cells.

account for higher osteogenic differentiation level of rADSCs. Nguyen et $\mathrm{al}^{22}$ displayed accelerated and enhanced osteoblastic differentiation of MSCs on 3D scaffold than 2D scaffold, which is consistent with our findings.

\section{PCR analysis}

RT-PCR analysis of osteogenic genes of rADSCs on the three scaffolds after osteogenic induction of 7 and 14 days is shown in Figure 7. Alp, Coll, and Ocn were biomarkers during osteogenic differentiation of stem cells. ${ }^{45} M s \times 2$ is the homeobox gene in osteoblast and is a direct gene target of BMP-2. ${ }^{46}$ The rADSCs on PP-B and 3D scaffolds exhibited a significantly higher level of Alp, Coll, and Ocn than rADSCs on PP scaffolds both after osteogenic induction of 7 and 14 days $(P<0.05)$. A higher upregulated gene level was observed in the $3 \mathrm{D}$ group than in the $\mathrm{PP}-\mathrm{B}$ group, except Msx2. Msx2 can be upregulated by BMP-2 and facilitate osteogenic differentiation. ${ }^{47}$ Higher concentration of BMP-2 in the PP-B group accounted for its higher Msx2 expression and enhanced differentiation of rADSCs. Studies reported that silk fibroin scaffold incorporated with BMP-2 greatly enhanced bone-related markers' expression level of
MSCs. ${ }^{48}$ BMP-2 is able to recruit stem cells and facilitate its osteoblastic differentiation. Shalumon et $\mathrm{al}^{33}$ fabricated core-shell nanofibrous scaffolds with BMP-2 in core and tested osteogenic markers of human bone marrow-derived MSCs (hMSCs) from 0 to 28 days. They found that BMP-2 released from the scaffolds played an important role in osteogenic differentiation. The rADSCs on 3D scaffold expressed the highest level of bone-related markers. Cooperation of BMP-2 and 3D structure may contribute to the increased osteogenic differentiation. Ocn was biomarker expressed in late stage in osteoblast differentiation. A higher Ocn expression was observed in 7 and 14 days in the 3D group. This indicated the maturation of osteogenic differentiation of rADSCs on 3D scaffolds. So, 3D scaffold was able to promote cell differentiation into osteogenic lineage. In conclusion, two main reasons were account for stronger differentiation ability in 3D group. First, 3D scaffold increased cell proliferation and enhanced cell-cell and cell-matrix interactions and this would exhibit a positive effect on osteogenic differentiation. ${ }^{21}$ Second, BMP-2 released from the scaffold and its 3D architecture was important in cell differentiation. 


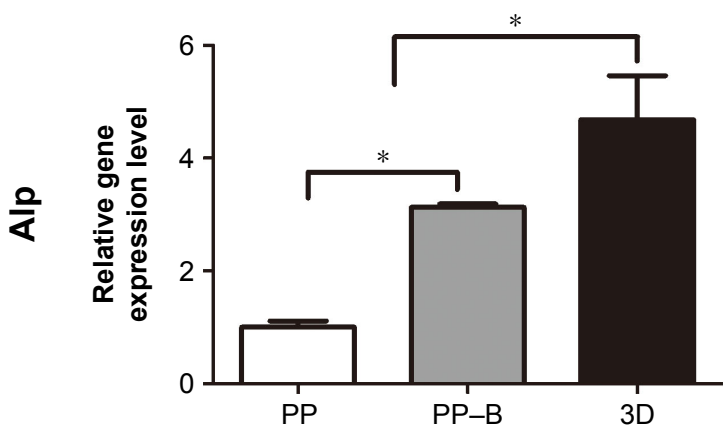

\section{7 days}
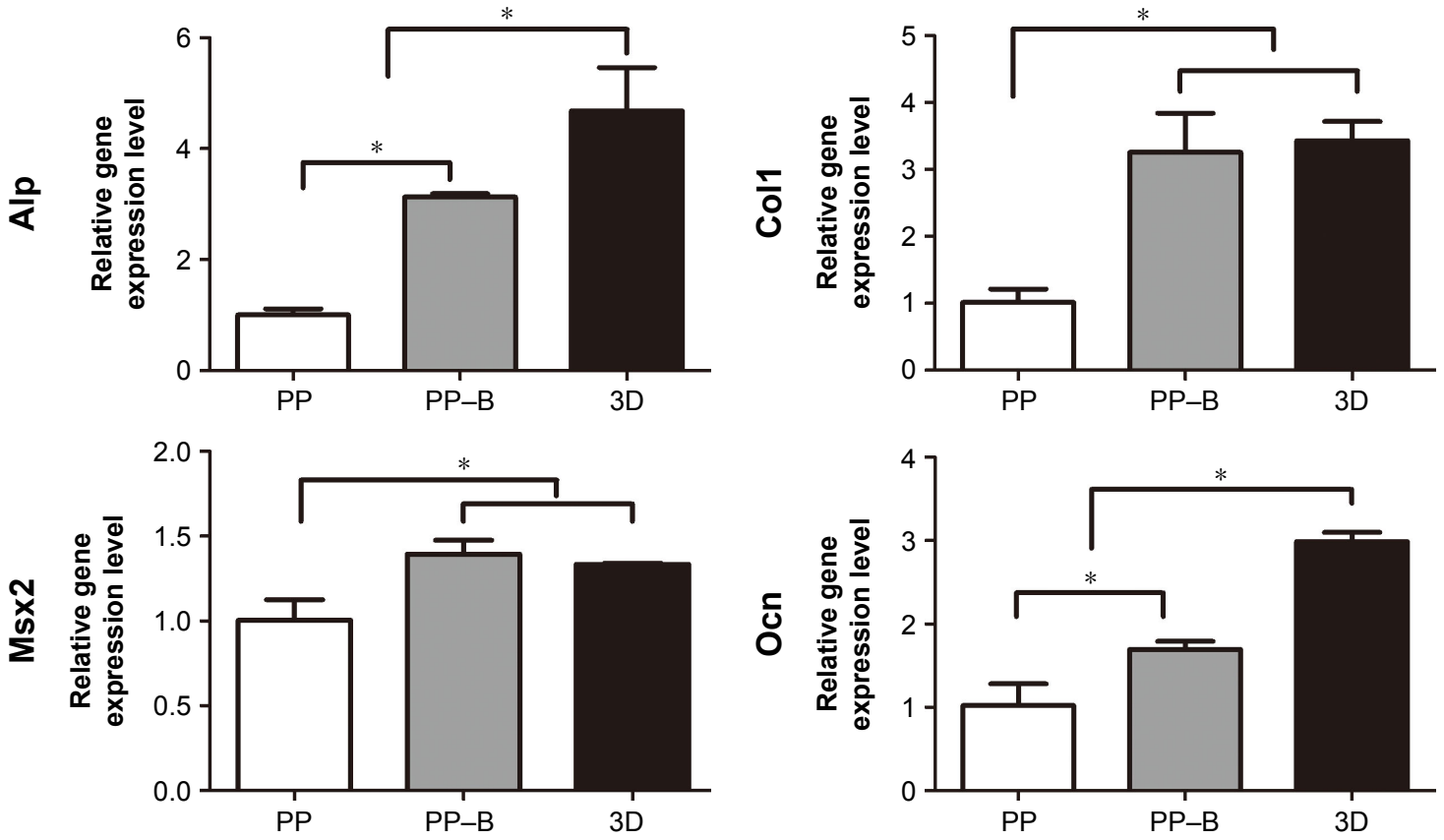

14 days
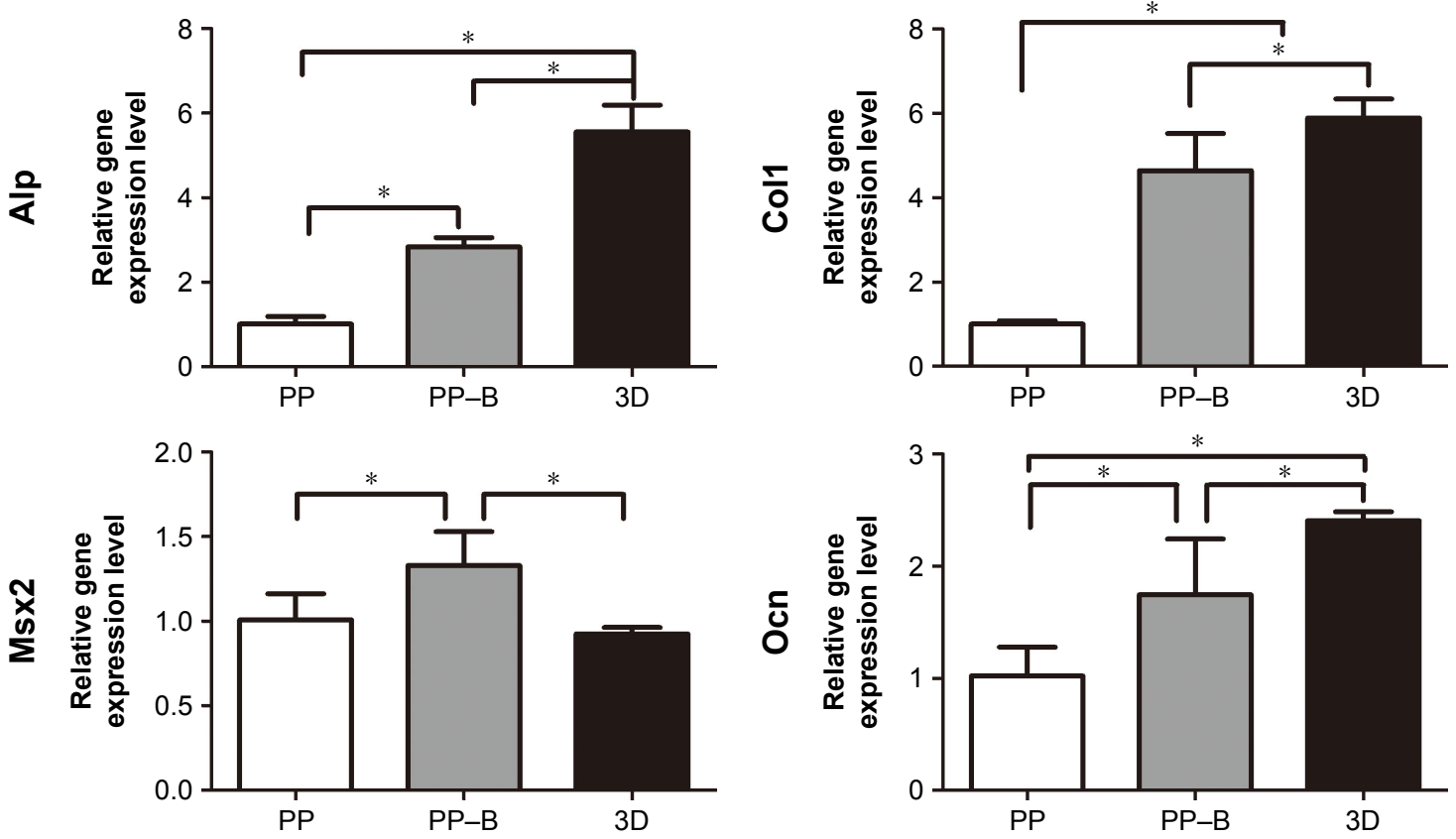

Figure 7 RT-PCR analysis of Alp, Coll, Msx2, and Ocn osteogenic gene expression levels of rADSCs on different scaffolds after osteogenic induction of 7 and I4 days $(* P<0.05)$.

Abbreviations: 3D, three dimension; PP, poly(lactide-co-glycolide)/polycaprolactone; PP-B, PP-bone morphogenetic protein-2; rADSCs, rat adipose-derived stem cells; RT-PCR, real-time quantitative reverse transcription polymerase chain reaction.

\section{Conclusion}

We prepared PP-B core-shell fibers with PP in shell and BMP-2 in core along with sustained release of BMP-2 up to 28 days. And 3D scaffolds were fabricated based on PP-B fibers with the TISA method. Better proliferation of rADSCs was found on PP-B and 3D scaffolds compared with PP group. 3D scaffold significantly enhanced osteogenic differentiation of rADSCs, followed by PP-B group. In summary, incorporation of BMP-2 in electrospun scaffold was able to promote the cell proliferation and osteogenic differentiation 
of rADSCs. The synergistic effects of 3D scaffold and osteoinductive BMP-2 significantly enhanced the tendency than $2 \mathrm{D}$ scaffolds in vitro. $3 \mathrm{D}$ porous material along with osteoinductive growth factor should be a promising scaffold in bone tissue engineering and dental medicine, especially for the reconstruction of bone defects.

\section{Acknowledgments}

This study was supported by grants from the State Key Research and Development Project, China (2016YFA020 1704/2016YFA0201700), and the Priority Academic Program Development of Jiangsu Higher Education Institutions (PAPD, 2014-37).

\section{Disclosure}

The authors report no conflicts of interest in this work.

\section{References}

1. Blatt S, Pabst AM, Schiegnitz E, et al. Early cell response of osteogenic cells on differently modified implant surfaces: sequences of cell proliferation, adherence and differentiation. J Craniomaxillofac Surg. 2018;46(3):453-460.

2. Fahimipour F, Dashtimoghadam E, Rasoulianboroujeni M, et al. Collagenous matrix supported by a 3D-printed scaffold for osteogenic differentiation of dental pulp cells. Dent Mater. 2018;34(2):209-220.

3. Boga JC, Miguel SP, de Melo-Diogo D, Mendonça AG, Louro RO, Correia IJ. In vitro characterization of 3D printed scaffolds aimed at bone tissue regeneration. Colloids Surf B Biointerfaces. 2018;165:207-218.

4. Mohammadi M, Mousavi SS, Alibolandi M, et al. Micro and nanotechnologies for bone regeneration: recent advances and emerging designs. J Control Release. 2018;274:35-55.

5. Wang Z, Lin M, Xie Q, et al. Electrospun silk fibroin/poly (lactide-

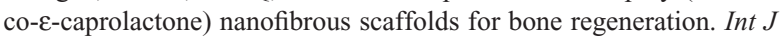
Nanomedicine. 2016;11:1483-1500.

6. Ortega Í, Ryan AJ, Deshpande P, MacNeil S, Claeyssens F. Combined microfabrication and electrospinning to produce 3-D architectures for corneal repair. Acta Biomater. 2013;9(3):5511-5520.

7. Lyu S, Huang C, Yang H, Zhang X. Electrospun fibers as a scaffolding platform for bone tissue repair. J Orthop Res. 2013;31(9):1382-1389.

8. Kennedy KM, Bhaw-Luximon A, Jhurry D. Cell-matrix mechanical interaction in electrospun polymeric scaffolds for tissue engineering: implications for scaffold design and performance. Acta Biomater. 2017; 50:41-55.

9. Ding Y, Li W, Müller T, et al. Electrospun polyhydroxybutyrate/poly ( $\varepsilon$-caprolactone)/58S sol-gel bioactive glass hybrid scaffolds with highly improved osteogenic potential for bone tissue engineering. ACS Appl Mater Interfaces. 2016;8(27):17098-17108.

10. Yang B, Zuo Y, Zou Q, et al. Effect of ultrafine poly ( $\varepsilon$-caprolactone) fibers on calcium phosphate cement: in vitro degradation and in vivo regeneration. Int J Nanomedicine. 2016;11:163-177.

11. Kim SE, Yun YP, Han YK, et al. Osteogenesis induction of periodontal ligament cells onto bone morphogenic protein-2 immobilized PCL fibers. Carbohydr Polym. 2014;99:700-709.

12. Bhattacharjee P, Naskar D, Maiti TK, et al. Non-mulberry silk fibroin grafted poly ( $\mathrm{C}$-caprolactone)/nano hydroxyapatite nanofibrous scaffold for dual growth factor delivery to promote bone regeneration. J Colloid Interface Sci. 2016;472:16-33.

13. El Bialy I, Jiskoot W, Nejadnik MR. Formulation, delivery and stability of bone morphogenetic proteins for effective bone regeneration. Pharm Res. 2017;34(6):1152-1170.
14. Moore NM, Lin NJ, Gallant ND, Becker ML. Synergistic enhancement of human bone marrow stromal cell proliferation and osteogenic differentiation on BMP-2-derived and RGD peptide concentration gradients. Acta Biomater. 2011;7(5):2091-2100.

15. Su Y, Su Q, Liu W, et al. Controlled release of bone morphogenetic protein 2 and dexamethasone loaded in core-shell PLLACL-collagen fibers for use in bone tissue engineering. Acta Biomater. 2012;8(2): 763-771.

16. Lu Y, Huang J, Yu G, et al. Coaxial electrospun fibers: applications in drug delivery and tissue engineering. Wiley Interdiscip Rev Nanomed Nanobiotechnol. 2016;8(5):654-677.

17. Khorshidi S, Solouk A, Mirzadeh H, et al. A review of key challenges of electrospun scaffolds for tissue-engineering applications. $J$ Tissue Eng Regen Med. 2016;10(9):715-738.

18. Zhong S, Zhang Y, Lim CT. Fabrication of large pores in electrospun nanofibrous scaffolds for cellular infiltration: a review. Tissue Eng Part B Rev. 2011;18(2):77-87.

19. Vashaghian M, Zandieh-Doulabi B, Roovers JP, Smit TH. Electrospun matrices for pelvic floor repair: effect of fiber diameter on mechanical properties and cell behavior. Tissue Eng Part A. 2016;22(23-24): $1305-1316$.

20. Jiang YC, Jiang L, Huang A, et al. Electrospun polycaprolactone/gelatin composites with enhanced cell-matrix interactions as blood vessel endothelial layer scaffolds. Mater Sci Eng C Mater Biol Appl. 2017;71: 901-908.

21. Sun B, Long YZ, Zhang HD, et al. Advances in three-dimensional nanofibrous macrostructures via electrospinning. Prog Polym Sci. 2014;39(5):862-890.

22. Nguyen LT, Liao S, Chan CK, Ramakrishna S. Enhanced osteogenic differentiation with 3D electrospun nanofibrous scaffolds. Nanomedicine. 2012;7(10):1561-1575.

23. Yao Q, Cosme JGL, Xu T, et al. Three dimensional electrospun PCL/ PLA blend nanofibrous scaffolds with significantly improved stem cells osteogenic differentiation and cranial bone formation. Biomaterials. 2017;115:115-127.

24. Lee JY, Son SJ, Son JS, Kang SS, Choi SH. Bone-healing capacity of PCL/PLGA/duck beak scaffold in critical bone defects in a rabbit model. Biomed Res Int. 2016;2016:2136215.

25. Hiep NT, Lee BT. Electro-spinning of PLGA/PCL blends for tissue engineering and their biocompatibility. J Mater Sci Mater Med. 2010;21(6):1969-1978.

26. Wei G, Li C, Fu Q, Xu Y, Li H. Preparation of PCL/silk fibroin/ collagen electrospun fiber for urethral reconstruction. Int Urol Nephrol. 2015;47(1):95-99.

27. Chen H, Qian Y, Xia Y, et al. Enhanced osteogenesis of ADSCs by the synergistic effect of aligned fibers containing collagen I. ACS Appl Mater Interfaces. 2016;8(43):29289-29297.

28. Dai J, Wang H, Liu G, Xu Z, Li F, Fang H. Dynamic compression and co-culture with nucleus pulposus cells promotes proliferation and differentiation of adipose-derived mesenchymal stem cells. J Biomech. 2014; 47(5):966-972.

29. Roskies MG, Fang D, Abdallah MN, et al. Three-dimensionally printed polyetherketoneketone scaffolds with mesenchymal stem cells for the reconstruction of critical-sized mandibular defects. Laryngoscope. 2017;127(11):E392-E398.

30. Xia L, Lin K, Jiang X, et al. Effect of nano-structured bioceramic surface on osteogenic differentiation of adipose derived stem cells. Biomaterials. 2014;35(30):8514-8527.

31. Xu T, Miszuk JM, Zhao Y, Sun H, Fong H. Electrospun polycaprolactone 3D nanofibrous scaffold with interconnected and hierarchically structured pores for bone tissue engineering. Adv Healthc Mater. 2015; 4(15):2238-2246.

32. Li N, Chen G, Liu J, et al. Effect of surface topography and bioactive properties on early adhesion and growth behavior of mouse preosteoblast MC3T3-E1 cells. ACS Appl Mater Interfaces. 2014;6(19): 17134-17143. 
33. Shalumon KT, Lai GJ, Chen CH, Chen JP. Modulation of bone-specific tissue regeneration by incorporating bone morphogenetic protein and controlling the shell thickness of silk fibroin/chitosan/nanohydroxyapatite core-shell nanofibrous membranes. ACS Appl Mater Interfaces. 2015;7(38):21170-21181.

34. Hsu YH, Lin CT, Yu YH, Chou YC, Liu SJ, Chan EC. Dual delivery of active antibactericidal agents and bone morphogenetic protein at sustainable high concentrations using biodegradable sheath-core-structured drug-eluting nanofibers. Int J Nanomedicine. 2016;11:3927-3937.

35. Sun F, Zhou H, Lee J. Various preparation methods of highly porous hydroxyapatite/polymer nanoscale biocomposites for bone regeneration. Acta Biomater. 2011;7(11):3813-3828.

36. Zhang W, Wray LS, Rnjak-Kovacina J, et al. Vascularization of hollow channel-modified porous silk scaffolds with endothelial cells for tissue regeneration. Biomaterials. 2015;56:68-77.

37. Ma T, Li Y, Yang ST, Kniss DA. Effects of pore size in 3-D fibrous matrix on human trophoblast tissue development. Biotechnol Bioeng. 2000;70(6):606-618

38. Fu C, Yang X, Tan S, Song L. Enhancing cell proliferation and osteogenic differentiation of MC3T3-E1 pre-osteoblasts by BMP-2 delivery in graphene oxide-incorporated PLGA/HA biodegradable microcarriers. Sci Rep. 2017;7(1):12549.

39. Driscoll MK, Sun X, Guven C, Fourkas JT, Losert W. Cellular contact guidance through dynamic sensing of nanotopography. ACS Nano. 2014; 8(4):3546-3555.

40. Hong JK, Bang JY, Xu G, et al. Thickness-controllable electrospun fibers promote tubular structure formation by endothelial progenitor cells. Int J Nanomedicine. 2015;10:1189-1200.

41. Kim MS, Kim GH. Three-dimensional electrospun polycaprolactone (PCL)/alginate hybrid composite scaffolds. Carbohydr Polym. 2014;114:213-221.
42. Yang J, Shi P, Tu M, et al. Bone morphogenetic proteins: relationship between molecular structure and their osteogenic activity. Food Sci Hum Wellness. 2014;3(3-4):127-135.

43. Chen D, Harris MA, Rossini G, et al. Bone morphogenetic protein 2 (BMP-2) enhances BMP-3, BMP-4, and bone cell differentiation marker gene expression during the induction of mineralized bone matrix formation in cultures of fetal rat calvarial osteoblasts. Calcif Tissue Int. 1997; 60(3):283-290.

44. Liu Y, Deng LZ, Sun HP, et al. Sustained dual release of placental growth factor-2 and bone morphogenic protein-2 from heparin-based nanocomplexes for direct osteogenesis. Int J Nanomedicine. 2016;11: 1147-1158.

45. Liao HT, Lee MY, Tsai WW, et al. Osteogenesis of adipose-derived stem cells on polycaprolactone- $\beta$-tricalcium phosphate scaffold fabricated via selective laser sintering and surface coating with collagen type I. J Tissue Eng Regen Med. 2016;10(10):E337-E353.

46. Cheng SL, Shao JS, Charlton-Kachigian N, Loewy AP, Towler DA. MSX2 promotes osteogenesis and suppresses adipogenic differentiation of multipotent mesenchymal progenitors. J Biol Chem. 2003; 278(46):45969-45977.

47. Matsubara T, Kida K, Yamaguchi A, et al. BMP2 regulates Osterix through Msx2 and Runx2 during osteoblast differentiation. J Biol Chem. 2008;283(43):29119-29125.

48. Li C, Vepari C, Jin HJ, Kim HJ, Kaplan DL. Electrospun silk-BMP-2 scaffolds for bone tissue engineering. Biomaterials. 2006;27(16) 3115-3124.
International Journal of Nanomedicine

\section{Publish your work in this journal}

The International Journal of Nanomedicine is an international, peerreviewed journal focusing on the application of nanotechnology in diagnostics, therapeutics, and drug delivery systems throughou the biomedical field. This journal is indexed on PubMed Central, MedLine, CAS, SciSearch $\AA$, Current Contents $₫ /$ Clinical Medicine,

\section{Dovepress}

Journal Citation Reports/Science Edition, EMBase, Scopus and the Elsevier Bibliographic databases. The manuscript management system is completely online and includes a very quick and fair peer-review system, which is all easy to use. Visit http://www.dovepress.com/ testimonials.php to read real quotes from published authors. 